\title{
On the Hierarchical Product of Graphs and the Generalized Binomial Tree
}

\author{
L. Barrière, F. Comellas, C. Dalfó and M.A. Fiol \\ Departament de Matemàtica Aplicada IV \\ Universitat Politècnica de Catalunya \\ $\{$ lali, comellas, cdalfo, fiol $\} @ m a 4 . u p c . e d u$
}

September 20, 2007

\begin{abstract}
In this paper we follow the study of the hierarchical product of graphs, an operation recently introduced in the context of networks. A well-known example of such a product is the binomial tree which is the (hierarchical) power of the complete graph on two vertices. An appealing property of this structure is that all the eigenvalues are distinct. Here we show how to obtain a graph with this property by applying the hierarchical product. In particular, we propose a generalization of the binomial tree and some of its main properties are studied.
\end{abstract}

Keywords: Graph, Hierarchical product, Binomial tree, Adjacency matrix, Eigenvalues, Eigenvectors.

AMS classification: $05 \mathrm{C} 50,05 \mathrm{C} 05$.

\section{Introduction}

Some classical graphs, modeling real-life complex networks, present a modular or hierarchical structure. This is the case, for instance, of networks with nodes having high degree, which are known as hubs [1]. These nodes usually play a critical role in the information flow of the system because many of the other nodes send and receive information through them. In [2] the authors introduced the hierarchical product of graphs which produces graphs with a strong (connectedness) hierarchy in their vertices. In fact, the obtained graphs turn out to be subgraphs of the Cartesian product of the corresponding factors. In particular, when each factor is the complete graph $K_{2}$, the resulting graph is a spanning tree of the hypercube. Some well-known properties of the Cartesian product, such as a reduced mean distance and diameter, simple routing algorithms and some optimal communication protocols are inherited by the hierarchical product.

On the other hand, the study of the spectrum of a graph is relevant for estimating important structural properties, which provide information on the topological and communication characteristics of the corresponding network $[4,8,9,10,13,14]$. Among these properties, which are usually very hard to obtain by other methods, we have edgeexpansion and node-expansion, bisection width, diameter, maximum cut, connectivity, and partitions [6].

In this paper we follow the study of the hierarchical product of graphs. A wellknown example of such a product is the hypertree $[2,3]$ or binomial tree $T_{m}[7]$ which is the (hierarchical) $m$-th power of the complete graph $K_{2}$. An appealing property of 
this structure is that all its eigenvalues are distinct. As it was shown in [15], this fact has some structural consequences, such as the Abelianity of the automorphism group. Indeed, we showed in [2] that the automorphism group of $T_{m}$ is the symmetric group $S_{2}$. This, together with the high degree of hierarchy of such trees, results in a number of nice properties for their spectra. Here we show how to obtain graphs with the above spectral property by applying the hierarchical product. In particular, we propose a generalization of the binomial tree, which we call $r$-adic ( $m$-dimensional) hypertree $T_{r}^{m}$ and some of its main properties are studied. This structure is characterized by two main features: it is a spanning subgraph of the $r$-dimensional mesh, and its vertices have a high degree of (connectedness) hierarchy. More precisely, because of the relationship satisfied by the characteristic polynomials of $T_{r}^{m}$ and $T_{r}^{m-1}$, every eigenvalue of the latter gives rise to $r$ eigenvalues of the former. Consequently, there is a strong relationship between the eigenvalues and the eigenvectors of the $r$-adic hypertrees of different dimensions.

\section{Basic properties of the hierarchical product}

For the basic concepts, notation and results about graphs, see for instance [5]. The hierarchical product of two graphs, introduced in [2], is defined as follows. Let $G_{i}=$ $\left(V_{i}, E_{i}\right)$ be two graphs with vertex sets $V_{i}, i=1,2$, having a distinguished or root vertex, labeled 0. The hierarchical product $G_{2} \sqcap G_{1}$ is the graph with vertices $x_{2} x_{1}, x_{i} \in V_{i}$, and edges $\left\{x_{2} x_{1}, y_{2} y_{1}\right\}$ where either $y_{2}=x_{2}$ and $y_{1} \sim x_{1}$ in $G_{1}$, or $y_{1}=x_{1}=0$ and $y_{2} \sim x_{2}$ in $G_{2}$.

Thus, $G_{2} \sqcap G_{1}$ has $\left|V_{2}\right|\left|V_{1}\right|$ vertices and $\left|E_{2}\right|+\left|V_{2}\right|\left|E_{1}\right|$ edges. Also, notice that $G_{2} \sqcap G_{1}$ is a (spanning) subgraph of the Cartesian (or direct) product $G_{2} \square G_{1}$. (This fact suggested us to use the notation " $\square$ " for our product since, whereas $K_{2} \square K_{2}$ is a square, $K_{2} \sqcap K_{2}$ is a path on four vertices.) Although the Cartesian product is both commutative and associative, the hierarchical product has only the second property, provided that the root vertices are chosen in the natural way (for instance, the root of $G_{2} \square G_{1}$ is chosen to be $\mathbf{0}=00$ ). This allows us to recursively define the hierarchical product of $N$ graphs $G_{i}$, $i=1,2, \ldots, N$, as $G_{N} \sqcap \cdots \sqcap G_{2} \sqcap G_{1}=\left(G_{N} \sqcap \cdots \sqcap G_{2}\right) \sqcap G_{1}$. As a consequence, since clearly $K_{1} \sqcap G=G \sqcap K_{1}=G$, the set of graphs with the binary operation $\sqcap$ is a semigroup with identity element $K_{1}$ (that is, a monoid). A simple consequence of the above is the following result.

Lemma 2.1 [2] Let $H=G_{N} \sqcap \cdots \sqcap G_{2} \sqcap G_{1}$. For a fixed string $\boldsymbol{z}$ of appropriate length (for instance $\boldsymbol{z}=\mathbf{0}=00 \ldots 0$ ), let $H\left\langle\boldsymbol{z} x_{k} \ldots x_{1}\right\rangle$ denote the subgraph of $H$ induced by the vertex set $\left\{\boldsymbol{z} x_{k} \ldots x_{1} \mid x_{i} \in V_{i}, 1 \leq i \leq k\right\}$. Let $H\left\langle x_{N} \ldots x_{k} \boldsymbol{z}\right\rangle$ be defined analogously. Then,

(a) $H\left\langle\boldsymbol{z} x_{k} \ldots x_{1}\right\rangle=G_{k} \sqcap \cdots \sqcap G_{1}$ for any fixed $\boldsymbol{z}$;

(b) $H\left\langle x_{N} \ldots x_{k} \mathbf{0}\right\rangle=G_{N} \sqcap \cdots \sqcap G_{k}$;

(c) $H\left\langle x_{N} \ldots x_{k} \boldsymbol{z}\right\rangle=m K_{1}$ (that is, a set of $m=n_{N} \cdots n_{k}$ singletons) for $\boldsymbol{z} \neq \mathbf{0}$, where $n_{i}=\left|V_{i}\right|, k \leq i \leq N$.

Concerning some algebraic properties of the hierarchical product, it was shown in [2] that, if $G_{i}$ has adjacency matrix $\boldsymbol{A}_{i}, i=1,2$, then, the adjacency matrix of its hierarchical product $H=G_{2} \sqcap G_{1}$ is (under some appropriate labeling of its vertices):

$$
\boldsymbol{A}_{H}=\boldsymbol{A}_{2} \otimes \boldsymbol{D}_{1}+\boldsymbol{I}_{2} \otimes \boldsymbol{A}_{1} \cong \boldsymbol{D}_{1} \otimes \boldsymbol{A}_{2}+\boldsymbol{A}_{1} \otimes \boldsymbol{I}_{2}
$$


where $\otimes$ stands for the Kronecker product, and $\boldsymbol{D}_{1}=\operatorname{diag}(1,0, \ldots, 0)$ and $\boldsymbol{I}_{2}$ (the identity matrix) have size $n_{1} \times n_{1}$ and $n_{2} \times n_{2}$, respectively. For instance, let $G$ be a graph of order $N$, and consider its hierarchical product $H=G \sqcap P_{r}$ by the path $P_{r}$ with $r$ vertices and adjacency matrix $\boldsymbol{A}_{r}$. Then, using (1),

$$
\boldsymbol{A}_{H}=\boldsymbol{D}_{1} \otimes \boldsymbol{A}_{G}+\boldsymbol{A}_{r} \otimes \boldsymbol{I}_{N}=\left(\begin{array}{cccccc}
\boldsymbol{A}_{G} & \boldsymbol{I}_{N} & \mathbf{0} & \cdots & \mathbf{0} & \mathbf{0} \\
\boldsymbol{I}_{N} & \mathbf{0} & \boldsymbol{I}_{N} & \cdots & \mathbf{0} & \mathbf{0} \\
\mathbf{0} & \boldsymbol{I}_{N} & \mathbf{0} & \cdots & \mathbf{0} & \mathbf{0} \\
\vdots & \vdots & \vdots & \ddots & \vdots & \vdots \\
\mathbf{0} & \mathbf{0} & \mathbf{0} & \cdots & \mathbf{0} & \boldsymbol{I}_{N} \\
\mathbf{0} & \mathbf{0} & \mathbf{0} & \cdots & \boldsymbol{I}_{N} & \mathbf{0}
\end{array}\right)
$$

which is an $r \times r$ matrix of $N \times N$ blocks.

From this result, and using the expression for computing the determiant of a block matrix [17], it is possible to compute the characteristic polynomial of a generic two-term product:

Theorem 2.2 [2] Let $G_{i}, i=1,2$, be two graphs on $n_{i}$ vertices, with adjacency matrix $\boldsymbol{A}_{i}$ and characteristic polynomial $\phi_{i}(x)$. Let $G_{1}$ have root vertex 0 and consider the graph $G_{1}^{*}=G_{1}-0$, with adjacency matrix $\boldsymbol{A}_{1}^{*}$ and characteristic polynomial $\phi_{1}^{*}$. Then, the characteristic polynomial $\phi_{H}(x)$ of the hierarchical product $H=G_{2} \sqcap G_{1}$ is:

$$
\phi_{H}(x)=\phi_{1}^{*}(x)^{n_{2}} \phi_{2}\left(\frac{\phi_{1}(x)}{\phi_{1}^{*}(x)}\right) .
$$

(See [16] for another approach to an equivalent result.)

\section{Spectral properties of the hierarchical product $G \sqcap P_{r}$}

In $[2,3]$, it was shown that the hypertree (or binomial tree) has all its eigenvalues distinct. In fact, this is a particular case of the following proposition, which shows how to obtain a graph with this spectral property by means of the hierarchical product.

First, recall that the characteristic polynomial $\phi_{r}$ of the path $P_{r}$ satisfies the recurrence

$$
\phi_{r}(x)=x \phi_{r-1}(x)-\phi_{r-2}(x) \quad(r \geq 2)
$$

starting from $\phi_{0}(x)=1$ and $\phi_{1}(x)=x$. Consequently, $\phi_{r}(x)=U_{r}\left(\frac{x}{2}\right)$, where $U_{r}$ stands for the $r$-degree Chebyshev polynomial of the second kind [18]. As these polynomials have zeros at $x_{k}=\cos \left(\frac{k \pi}{r+1}\right)$, the eigenvalues of $P_{r}$ can be written as

$$
\lambda_{k}=-2 \cos \left(\frac{(k+1) \pi}{r+1}\right), \quad k=0,1, \ldots, r-1,
$$

with $\lambda_{0}<\lambda_{1}<\cdots<\lambda_{r-1}$ and $\lambda_{k}=-\lambda_{r-1-k}$. See, for instance, $[4,8]$.

Proposition 3.1 Let $G$ be a graph on $n$ vertices with all distinct eigenvalues, denoted $\lambda_{1}<\lambda_{2}<\cdots<\lambda_{n}$. Then, all the eigenvalues of the hierarchical product $H=G \sqcap P_{r}$, on $n r$ vertices, are also different, provided that the root of the path $P_{r}$ is chosen to be one of the two vertices of degree one. Moreover, every eigenvalue $\lambda_{i}$ of $G$ gives rise to $r$ eigenvalues of $H, \lambda_{0 i}<\lambda_{1 i}<\cdots<\lambda_{(r-1) i}$, which are the solutions of the equation

$$
\phi_{r}(x)-\lambda_{i} \phi_{r-1}(x)=0,
$$

such that $\lambda_{i}>\lambda_{j}$ implies $\lambda_{h i}>\lambda_{h j}$. 
Proof. With the above notation and using (3), the characteristic polynomial of $H$ is

$$
\phi_{H}(x)=\phi_{r-1}(x)^{n} \phi_{G}\left(\frac{\phi_{r}(x)}{\phi_{r-1}(x)}\right)
$$

since the root of $P_{r}$ is chosen to be one of the vertices of degree 1 and, thus, $P_{r}^{*}=P_{r}-0=$ $P_{r-1}$ and $\phi_{r}^{*}(x)=\phi_{r-1}(x)$.

Then, we have the following implications:

$$
\lambda \in \operatorname{sp} H \Leftrightarrow \phi_{H}(\lambda)=0 \Leftrightarrow \Phi_{r}(\lambda) \in \operatorname{sp} G
$$

where $\Phi_{r}(\lambda):=\frac{\phi_{r}(\lambda)}{\phi_{r-1}(\lambda)}$. (Note that the zeros of $\phi_{r-1}(x)$ are not zeros of $\phi_{H}(x)$ because $\phi_{G}(x)$ is a polynomial of degree $n$.) Therefore, every $\lambda_{i} \in \operatorname{sp} G$ gives rise to $r$ eigenvalues of $H$, denoted by $\lambda_{h i}, h=0,1, \ldots, r-1$, which are the solutions of the equation $\Phi_{r}(x)=\lambda_{i}$ corresponding to (5). In order to study such solutions we need to know the behavior of $\Phi_{r}(x)$. First, let us see that this (rational) function is strictly increasing on its domain. Indeed, by (4) we have that $\Phi_{r}$ satisfies the following recurrence relation

$$
\Phi_{r}(x)=\frac{\phi_{r}(x)}{\phi_{r-1}(x)}=x-\frac{\phi_{r-2}(x)}{\phi_{r-1}(x)}=x-\frac{1}{\Phi_{r-1}(x)} \quad(r>2)
$$

with $\Phi_{1}(x)=\frac{\phi_{1}(x)}{\phi_{0}(x)}=x$ strictly increasing. By using induction, the fact that $\Phi_{r-1}(x)$ is strictly increasing implies that $\Phi_{r}(x)$ also is, because it is the sum of two strictly increasing functions.

Moreover, the function $\Phi_{r}(x)$ has the same zeros as $\phi_{r}$, that is, $x_{k}=-2 \cos \left(\frac{(k+1) \pi}{r+1}\right)$, $k=0,1, \ldots, r-1$, and asymptotes at $\widetilde{x}_{\ell}=-2 \cos \left(\frac{\ell \pi}{r}\right), \ell=1, \ldots, r-1$, which interlace the zeros, that is,

$$
x_{0}<\widetilde{x}_{1}<x_{1}<\widetilde{x}_{2}<x_{2}<\cdots<x_{r-2}<\widetilde{x}_{r-1}<x_{r-1} .
$$

(A well-known property of any sequence of orthogonal polynomials $[12,18]$.)

Consequently, if $r$ is even, then every $\lambda_{i} \in \operatorname{sp} G$ yields, in $H, \frac{r}{2}$ positive eigenvalues,

$$
0<\lambda_{\frac{r}{2}, i}<\lambda_{\frac{r}{2}+1, i}<\cdots<\lambda_{r-1, i}
$$

and $\frac{r}{2}$ negative eigenvalues

$$
\lambda_{0, i}<\lambda_{1, i}<\cdots<\lambda_{\frac{r}{2}-1, i}<0 .
$$

On the other hand, if $r$ is odd, then every positive (respectively, negative) eigenvalue $\lambda_{i} \in \operatorname{sp} G$ yields, in $H, \frac{r+1}{2}$ (respectively, $\frac{r-1}{2}$ ) positive eigenvalues, and $\frac{r-1}{2}$ (respectively, $\frac{r+1}{2}$ ) negative eigenvalues. Moreover, if $0 \in \operatorname{sp} G$, then we get in $H$ the eigenvalue $0, \frac{r-1}{2}$ positive eigenvalues and the same number of negative eigenvalues. Finally, the condition on the ordering of the obtained eigenvalues is due to the increasing character of the function. (As an example, Fig. 1 shows how each eigenvalue of $G$ yields three eigenvalues of $G \sqcap P_{3}$.)

Being more precise, notice that the eigenvalues of $H$ are distributed within the intervals bounded by the asymptotes in the following way: for every $i_{0}, i_{1}, \ldots, i_{r-1}$ in $\{1,2, \ldots, n\}$ we have

$$
\lambda_{0 i_{0}}<\widetilde{x}_{1}<\lambda_{1 i_{1}}<\widetilde{x}_{2}<\lambda_{2 i_{2}}<\cdots<\lambda_{(r-2) i_{r-2}}<\widetilde{x}_{r-1}<\lambda_{(r-1) i_{r-1}}
$$




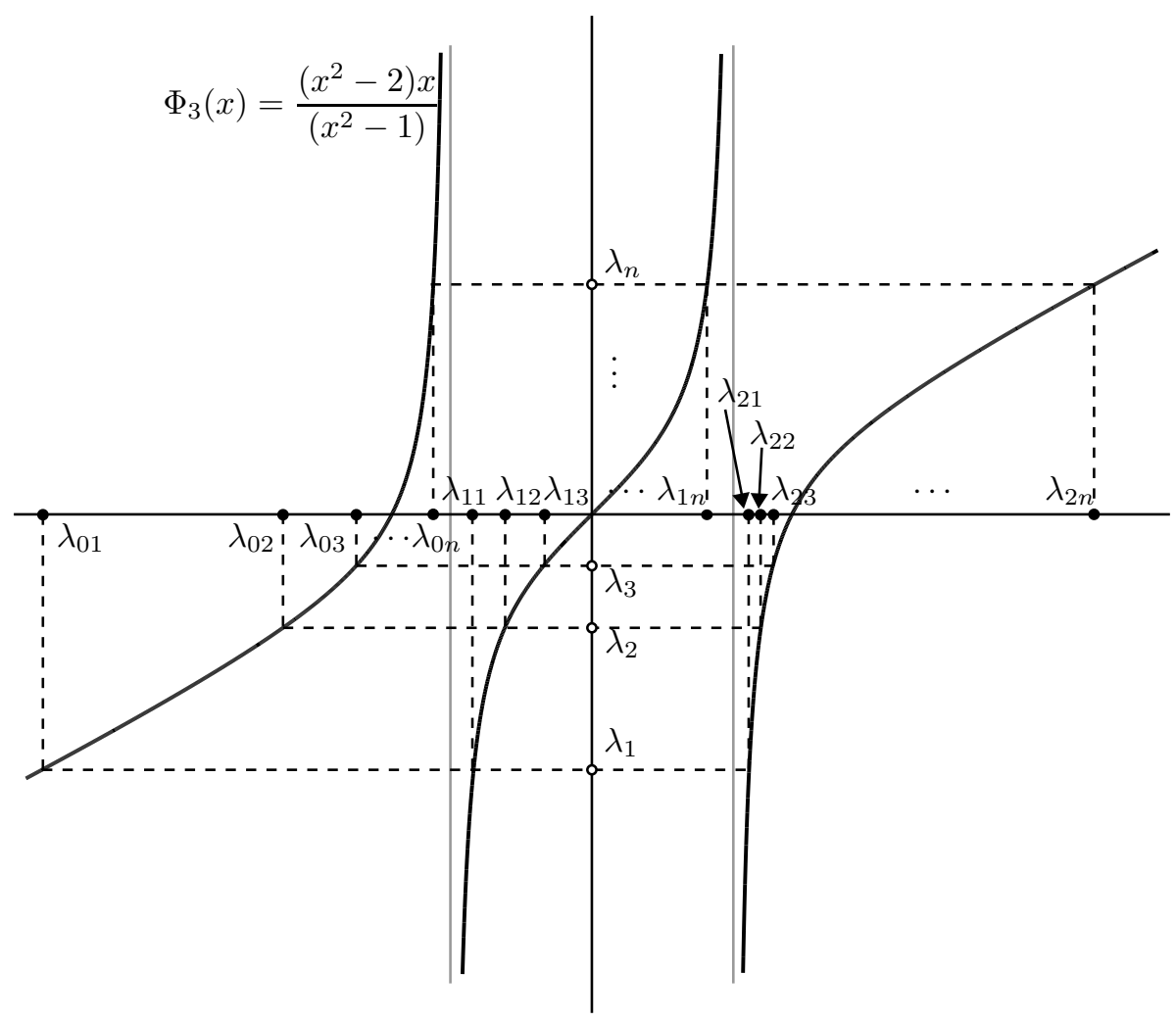

Figure 1: From $\circ \in \operatorname{sp} G$ to $\bullet \in \operatorname{sp}\left(G \sqcap P_{3}\right)$ through $\Phi_{3}(x)=\frac{\phi_{3}(x)}{\phi_{2}(x)}=\frac{\left(x^{2}-2\right) x}{x^{2}-1}$.

(compare with (8)). In other words, there exist functions $f_{h}, h=0,1, \ldots, r-1$, such that, when evaluated on each $\lambda_{i} \in \operatorname{sp} G$, give the eigenvalues $\lambda_{h i} \in \operatorname{sp}\left(G \sqcap P_{r}\right)$ which are the solutions of (5) satisfying

$$
\begin{aligned}
\lambda_{0 i} & =f_{0}\left(\lambda_{i}\right) \in I_{0}=\left(-\infty, \widetilde{x}_{1}\right), \\
\lambda_{h i} & =f_{h}\left(\lambda_{i}\right) \in I_{h}=\left(\widetilde{x}_{h}, \widetilde{x}_{h+1}\right), \quad h=1,2, \ldots, r-2, \\
\lambda_{(r-1) i} & =f_{r-1}\left(\lambda_{i}\right) \in I_{r-1}=\left(\widetilde{x}_{r-1}, \infty\right) .
\end{aligned}
$$

For instance, when $r=2$ the equation to be solved is $\Phi_{2}(x)=\frac{x^{2}-1}{x}=\lambda_{i}$ and, then, the above functions are

$$
f_{0}\left(\lambda_{i}\right)=\frac{\lambda_{i}-\sqrt{\lambda_{i}^{2}+4}}{2}<0, \quad f_{1}\left(\lambda_{i}\right)=\frac{\lambda_{i}+\sqrt{\lambda_{i}^{2}+4}}{2}>0 .
$$

Another simple consequence of the above proposition is the following result.

Corollary 3.2 If $\lambda$ is a non-zero eigenvalue of $G$ and $\frac{1}{\lambda}$ is an eigenvalue of $P_{r+1}$, then $\lambda$ is also an eigenvalue of $G \sqcap P_{r}$.

Proof. From the hypothesis $\phi_{r+1}\left(\frac{1}{\lambda}\right)=0$ and, by using (4), we have that

$$
\frac{1}{\lambda} \phi_{r}\left(\frac{1}{\lambda}\right)-\phi_{r-1}\left(\frac{1}{\lambda}\right)=0 .
$$

Then, $\frac{1}{\lambda}$ is a solution of (5), with $\lambda_{i}=\frac{1}{\lambda}$, and Proposition 3.1 gives the result.

As an example, $G=P_{4}$ has the eigenvalues $\lambda_{0}<\lambda_{1}<\lambda_{2}<\lambda_{3}$ satisfying $\lambda_{0}=\lambda_{1}^{-1}=$ $-2 \cos \left(\frac{\pi}{5}\right)=-\frac{1+\sqrt{5}}{2}$ and $\lambda_{3}=\lambda_{2}^{-1}=2 \cos \left(\frac{\pi}{5}\right)=\frac{1+\sqrt{5}}{2}$. Then, from the above corollary $\operatorname{sp} P_{4} \subset \operatorname{sp}\left(P_{4} \sqcap P_{3}\right)$. 
Another approach for finding the eigenvalues of $G \sqcap P_{r}$ is through their respective eigenvectors, as the next result shows.

Proposition 3.3 Let $\lambda$ be an eigenvalue of $G$ with corresponding eigenvector $\boldsymbol{u}$. Let us consider the $r \times r$ (symmetric) matrix

$$
\boldsymbol{A}(\lambda)=\left(\begin{array}{cccccc}
\lambda & 1 & 0 & \cdots & 0 & 0 \\
1 & 0 & 1 & \cdots & 0 & 0 \\
0 & 1 & 0 & \cdots & 0 & 0 \\
\vdots & \vdots & \vdots & \ddots & \vdots & \vdots \\
0 & 0 & 0 & \cdots & 0 & 1 \\
0 & 0 & 0 & \cdots & 1 & 0
\end{array}\right)
$$

with eigenvalues $\lambda_{0}, \lambda_{1}, \ldots, \lambda_{r-1}$ and corresponding eigenvectors $\boldsymbol{w}_{0}, \boldsymbol{w}_{1}, \ldots, \boldsymbol{w}_{r-1}$. Then, the hierarchical product $H=G \sqcap P_{r}$ has the same eigenvalues with corresponding eigenvectors $\boldsymbol{w}_{0} \otimes \boldsymbol{u}, \boldsymbol{w}_{1} \otimes \boldsymbol{u}, \ldots, \boldsymbol{w}_{r-1} \otimes \boldsymbol{u}$ (column vectors must be seen as $r \times 1$ matrices).

Proof. From the hypothesis, $\boldsymbol{A}_{G} \boldsymbol{u}=\lambda \boldsymbol{u}$. Moreover, for every $h, 0 \leq h \leq r-1$, let $\boldsymbol{w}_{h}=\left(w_{h(r-1)}, w_{h(r-2)}, \ldots, w_{h 0}\right)^{\top}$ be the eigenvector of $\boldsymbol{A}(\lambda)$, satisfying $\boldsymbol{A}(\lambda) \overline{\boldsymbol{w}}_{h}=\lambda_{h} \boldsymbol{w}_{h}$, which corresponds to the equations

$$
\begin{aligned}
\lambda w_{h(r-1)}+w_{h(r-2)} & =\lambda_{h} w_{h(r-1)} \\
w_{h(r-1)}+w_{h(r-3)} & =\lambda_{h} w_{h(r-2)} \\
w_{h(r-2)}+w_{h(r-4)} & =\lambda_{h} w_{h(r-3)} \\
& \vdots \\
w_{h 2}+w_{h 0} & =\lambda_{h} w_{h 1} \\
w_{h 1} & =\lambda_{h} w_{h 0} .
\end{aligned}
$$

Then,

$$
\begin{aligned}
\boldsymbol{A}_{H}\left(\boldsymbol{w}_{h} \otimes \boldsymbol{u}\right)= & \left(\begin{array}{cccccc}
\boldsymbol{A}_{G} & \boldsymbol{I}_{N} & \mathbf{0} & \cdots & \mathbf{0} & \mathbf{0} \\
\boldsymbol{I}_{N} & \mathbf{0} & \boldsymbol{I}_{N} & \cdots & \mathbf{0} & \mathbf{0} \\
\mathbf{0} & \boldsymbol{I}_{N} & \mathbf{0} & \cdots & \mathbf{0} & \mathbf{0} \\
\vdots & \vdots & \vdots & \ddots & \vdots & \vdots \\
\mathbf{0} & \mathbf{0} & \mathbf{0} & \cdots & \mathbf{0} & \boldsymbol{I}_{N} \\
\mathbf{0} & \mathbf{0} & \mathbf{0} & \cdots & \boldsymbol{I}_{N} & \mathbf{0}
\end{array}\right)\left(\begin{array}{c}
w_{h(r-1)} \boldsymbol{u} \\
w_{h(r-2)} \boldsymbol{u} \\
w_{h(r-3)} \boldsymbol{u} \\
\vdots \\
w_{h 1} \boldsymbol{u} \\
w_{h 0} \boldsymbol{u}
\end{array}\right) \\
= & \left(\begin{array}{c}
w_{h(r-1)} \lambda \boldsymbol{u}+w_{h(r-2)} \boldsymbol{u} \\
w_{h(r-1)} \boldsymbol{u}+w_{h(r-3)} \boldsymbol{u} \\
w_{h(r-2)} \boldsymbol{u}+w_{h(r-4)} \boldsymbol{u} \\
\vdots \\
w_{h 2} \boldsymbol{u}+w_{h 0} \boldsymbol{u} \\
w_{h 1} \boldsymbol{u}
\end{array}\right)=\lambda_{h}\left(\begin{array}{c}
w_{h(r-1)} \boldsymbol{u} \\
w_{h(r-2)} \boldsymbol{u} \\
w_{h(r-3)} \boldsymbol{u} \\
\vdots \\
w_{h 1} \boldsymbol{u} \\
w_{h 0} \boldsymbol{u}
\end{array}\right)=\lambda_{h}\left(\boldsymbol{w}_{h} \otimes \boldsymbol{u}\right),
\end{aligned}
$$

as claimed.

Note that, from the above result, the characteristic polynomial of $\boldsymbol{A}(\lambda)$ coincides with the polynomial in (5), that is,

$$
\phi_{\boldsymbol{A}(\lambda)}(x)=\phi_{r}(x)-\lambda \phi_{r-1}(x) .
$$


Notice also that, as $\boldsymbol{A}(\lambda)$ diagonalizes, the eigenvectors $\boldsymbol{w}_{0}, \boldsymbol{w}_{1}, \ldots, \boldsymbol{w}_{r-1}$ are linearly independent and so are the eigenvectors $\boldsymbol{w}_{0} \otimes \boldsymbol{u}, \boldsymbol{w}_{1} \otimes \boldsymbol{u}, \ldots, \boldsymbol{w}_{r-1} \otimes \boldsymbol{u}$. In fact, for every eigenvalue $\lambda_{h}$ of $\boldsymbol{A}(\lambda)$, its corresponding eigenvector can be computed by evaluating at $\lambda_{h}$ the characteristic polynomial $\phi_{i}$ of the path $P_{i}$, for $i=r-1, r-2, \ldots, 0$, as the next lemma shows.

Lemma 3.4 If the matrix $\boldsymbol{A}(\lambda)$ has the eigenvalue $\lambda_{h}$, then its corresponding eigenvector is

$$
\boldsymbol{w}_{h}=\left(w_{h(r-1)}, w_{h(r-2)}, \ldots, w_{h 0}\right)^{\top}=\left(\phi_{r-1}\left(\lambda_{h}\right), \phi_{r-2}\left(\lambda_{h}\right), \ldots, \phi_{0}\left(\lambda_{h}\right)\right)^{\top} .
$$

Proof. Substituting $w_{h i}$ by $\phi_{i}\left(\lambda_{h}\right)$, for $i=r-1, \ldots, 0$, into (11), we are lead to prove the following equations:

$$
\begin{aligned}
\lambda \phi_{r-1}\left(\lambda_{h}\right)+\phi_{r-2}\left(\lambda_{h}\right) & =\lambda_{h} \phi_{r-1}\left(\lambda_{h}\right) \\
\phi_{r-1}\left(\lambda_{h}\right)+\phi_{r-3}\left(\lambda_{h}\right) & =\lambda_{h} \phi_{r-2}\left(\lambda_{h}\right) \\
\phi_{r-2}\left(\lambda_{h}\right)+\phi_{r-4}\left(\lambda_{h}\right) & =\lambda_{h} \phi_{r-3}\left(\lambda_{h}\right) \\
& \vdots \\
\phi_{2}\left(\lambda_{h}\right)+\phi_{0}\left(\lambda_{h}\right) & =\lambda_{h} \phi_{1}\left(\lambda_{h}\right) \\
\phi_{1}\left(\lambda_{h}\right) & =\lambda_{h} \phi_{0}\left(\lambda_{h}\right) .
\end{aligned}
$$

The first equation holds since, by using (4),

$$
\lambda_{h} \phi_{r-1}\left(\lambda_{h}\right)-\phi_{r-2}\left(\lambda_{h}\right)-\lambda \phi_{r-1}\left(\lambda_{h}\right)=\phi_{r}\left(\lambda_{h}\right)-\lambda \phi_{r-1}\left(\lambda_{h}\right)=\phi_{\boldsymbol{A}(\lambda)}\left(\lambda_{h}\right)=0
$$

Also, the following $r-2$ equations correspond to (4) with $x=\lambda_{h}$, whereas the last one holds since $\phi_{0}(x)=1$ and $\phi_{1}(x)=x$.

\section{The $r$-adic hypertree}

As a generalization of the hypertree $T_{m}=K_{2}^{m}=K_{2} \sqcap K_{2} \sqcap \stackrel{m}{\cdots} \sqcap K_{2}$ (defined in [2]), we propose the $r$-adic hypertree (of dimension $m$ ) denoted and defined as

$$
T_{r}^{m}:=P_{r}^{m}=P_{r} \sqcap P_{r} \sqcap \stackrel{m}{\cdots} \sqcap P_{r},
$$

where $P_{r}$ is the path on $r$ vertices (and with length $r-1$ ). By convention, $T_{r}^{0}=K_{1}$. For $r=2$, notice that $T_{2}^{m}$ corresponds to the above hypertree (or binomial tree) $T_{m}$. As an example, Fig. 2 shows the 3 -adic hypertree $T_{3}^{3}$.

\subsection{Basic properties of the $r$-adic hypertree}

Some basic properties of the $r$-adic hypertree are:

- The order and the size are $\left|T_{r}^{m}\right|=r^{m}$ and $\left\|T_{r}^{m}\right\|=r^{m}-1$.

- $T_{r}^{m}=T_{r}^{m-1} \sqcap P_{r}$ (since the hierarchical product is associative).

- $T_{r}^{m}$ is a spanning subgraph of the $m$-dimensional mesh $P_{r} \square P_{r} \square \stackrel{m}{\cdots} \square P_{r}$.

- Let $E$ be the edge set $\{\{0 \mathbf{0}, 1 \mathbf{0}\},\{1 \mathbf{0}, 20\}, \ldots,\{(r-2) \mathbf{0},(r-1) \mathbf{0}\}\}$. Then, $T_{r}^{m}-E$ is isomorphic to the disjoint union of $r$ copies of $T_{r}^{m-1}$. In fact, such copies of $T_{r}^{m-1}$ are the subgraphs of $T_{r}^{m}$ induced by the vertex sets

$$
V_{\alpha}=\left\{\alpha \boldsymbol{w} \mid \boldsymbol{w} \in \mathbb{Z}_{r}^{m-1}\right\},
$$

for $0 \leq \alpha \leq r-1$. (Lemma 2.1(a) with $\boldsymbol{z}=\alpha$.) 


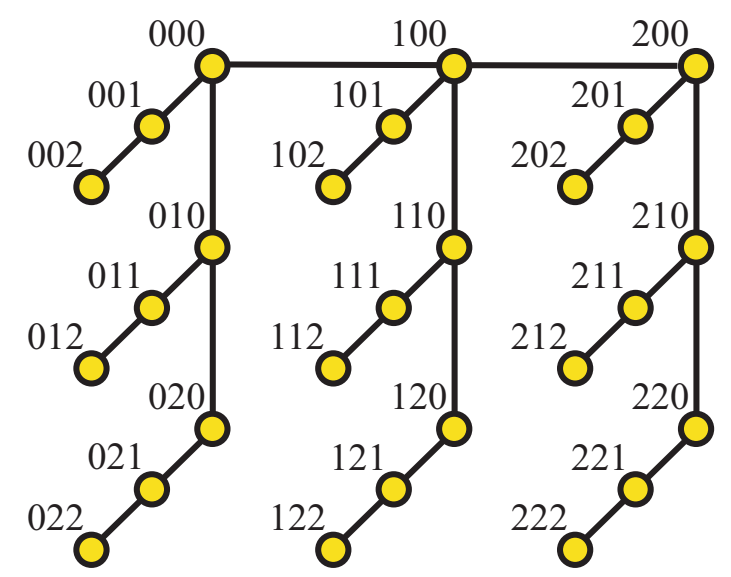

Figure 2: The 3-adic 3-dim hypertree $T_{3}^{3}$.

- The degree distribution of the vertices of $T_{r}^{m}$ is the following:

1. $\delta(\alpha \mathbf{0})=m+1$, for $\alpha \neq 0, r-1$;

2. $\delta(0 \mathbf{0})=\delta((r-1) \mathbf{0})=m$;

3. $\delta(\boldsymbol{w} \alpha 0 \stackrel{i-1}{-1} 0)=i+1$, for $\alpha \neq 0, r-1$, and $1 \leq i \leq m-1$;

4. $\delta(\boldsymbol{w}(r-1) 0 \stackrel{i-1}{-} 0)=i$, for $1 \leq i \leq m-1$.

This gives:

- $r-2$ vertices of degree $m+1$ (case 1$)$;

- $r(r-2)+2$ vertices of degree $m$ (case 2 and case 3 with $i=m-1$ );

- $r^{j}+r^{j+1}(r-2)$ vertices of degree $m-j$, for $1 \leq j \leq m-2$ (case 3 with $i=m-j-1$ and case 4 with $i=m-j$ );

- $r^{m-1}$ vertices of degree 1 (case 4 with $i=1$ ).

- Let us consider in $T_{r}^{m}$ a generic vertex $\boldsymbol{u}=u_{m-1} u_{m-2} \ldots u_{0}$, where $u_{i} \in \mathbb{Z}_{r}$. Then, its distance to the root $\mathbf{0}=00 \ldots 0$ is

$$
\operatorname{dist}(\mathbf{0}, \boldsymbol{u})=\sum_{i=0}^{m-1} u_{i} .
$$

- In order to find the (shortest path) routing $\boldsymbol{u} \rightarrow \mathbf{0}$, we successively decrease to 0 every digit of $\boldsymbol{u}$, from the right to the left. For example, if $\boldsymbol{u}=3102 \in V\left(T_{4}^{4}\right)$, its shortest path to $\mathbf{0}$ is

$$
\boldsymbol{u}=3102 \rightarrow 3101 \rightarrow 3100 \rightarrow 3000 \rightarrow 2000 \rightarrow 1000 \rightarrow 0000=\mathbf{0} .
$$

This path is unique, as $T_{r}^{m}$ is a tree.

- The eccentricity of the root is

$$
\operatorname{ecc}(\mathbf{0})=(r-1) m,
$$

with only one vertex at maximum distance, namely $(r-1)(r-1) \ldots(r-1)$. 


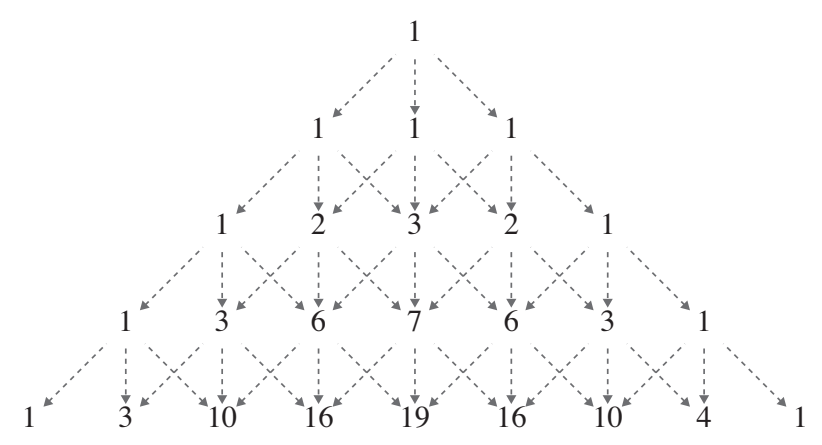

Figure 3: The recurence property of $N_{3}^{m}$ shown as a Pascal-like triangle.

- The radius (minimum eccentricity) of $T_{r}^{m}$ is

$$
r\left(T_{r}^{m}\right)=(r-1)(m-1)+\left\lfloor\frac{r}{2}\right\rfloor .
$$

If $r$ is odd, there is exactly one central vertex, $\frac{r-1}{2} 0 \ldots 0$, with two vertices at maximum distance, $0(r-1) \ldots(r-1)$ and $(r-1)(r-1) \ldots(r-1)$.

If $r$ is even, there are two central vertices:

- $\frac{r-2}{2} 0 \ldots 0$, with only the vertex $(r-1)(r-1) \ldots(r-1)$ at maximum distance, and

- $\frac{r}{2} 0 \ldots 0$, with only the vertex $0(r-1) \ldots(r-1)$ at maximum distance.

- As $T_{r}^{m}=P_{r} \sqcap T_{r}^{m-1}$, the diameter of $T_{r}^{m}$ satisfies the following recurrence:

$$
D\left(T_{r}^{m}\right)=2(r-1)+D\left(T_{r}^{m-1}\right)
$$

which, as $D\left(T_{r}^{1}\right)=D\left(P_{r}\right)=r-1$, yields

$$
D\left(T_{r}^{m}\right)=(r-1)(2 m-1) .
$$

Let $\Gamma_{r}^{m}(k)$ denote the set of vertices in $T_{r}^{m}$ which are at distance $k$ from the root $\mathbf{0}$, with cardinality $N_{r}^{m}(k):=\left|\Gamma_{r}^{m}(k)\right|$. For $r=2$, it is known that $N_{2}^{m}(k)=\left(\begin{array}{c}m \\ k\end{array}\right)$, as the vertices at distance $k$ from $\mathbf{0}$ have exactly $k$ 1's. In general, $N_{r}^{m}(k)$ is the number of words with length $m$ over the alphabet of $r$ symbols $\{0,1, \ldots, r-1\}$, such that they add up to $k$. Then, the following result can be seen as a generalization of the corresponding properties for the binomial tree.

Proposition 4.1 The number $N_{r}^{m}(k)$ of vertices at distance $k$ from the root $\mathbf{0}$ in the $r$-adic hypertree $T_{r}^{m}$ satisfies the following:

(a) Symmetry property: Let $\varepsilon=\operatorname{ecc}(\mathbf{0})$. Then,

$$
N_{r}^{m}(k)=N_{r}^{m}(\varepsilon-k),
$$

for every $0 \leq k \leq \varepsilon$.

(b) Recurrence property:

$$
N_{r}^{m}(k)= \begin{cases}N_{r}^{m-1}(0)+N_{r}^{m-1}(1)+\cdots+N_{r}^{m-1}(k) & \text { if } k<r-1, \\ N_{r}^{m-1}(k-r+1)+N_{r}^{m-1}(k-r+2)+\cdots+N_{r}^{m-1}(k) & \text { if } r-1 \leq k<\frac{\varepsilon}{2} .\end{cases}
$$


Proof. (a) The application $\psi: V\left(T_{r}^{m}\right) \rightarrow V\left(T_{r}^{m}\right)$ defined by

$$
\psi(\boldsymbol{u})=\psi\left(u_{m-1} u_{m-2} \ldots u_{0}\right)=\overline{\boldsymbol{u}}=\bar{u}_{m-1} \bar{u}_{m-2} \ldots \bar{u}_{0},
$$

where $\bar{u}_{i}:=r-1-u_{i}$, is a bijection from $V\left(T_{r}^{m}\right)$ into itself, and it maps each vertex $\boldsymbol{u}$ at distance $k$ (from $\mathbf{0}$ ) into the vertex $\overline{\boldsymbol{u}}$ at distance $\varepsilon-k$ (also from $\mathbf{0}$ ). Indeed, if $\operatorname{dist}(\mathbf{0}, \boldsymbol{u})=k$, then

$$
\operatorname{dist}(\mathbf{0}, \overline{\boldsymbol{u}})=\sum_{i=0}^{m-1} \bar{u}_{i}=\sum_{i=0}^{m-1}\left(r-1-u_{i}\right)=m(r-1)-k=\varepsilon-k
$$

where we have used (14). Therefore, $\psi$ is a bijection from $\Gamma_{r}^{m}(k)$ to $\Gamma_{r}^{m}(\varepsilon-k)$.

(b) Let us assume that $r-1 \leq k<\frac{\varepsilon}{2}$ (the proof of the other case being basically the same). Consider the set $\Gamma_{r}^{m}(k)$ partitioned into $r$ subsets obtained by fixing the value $u_{0}=j, 0 \leq j \leq r-1$. That is,

$$
\Gamma_{r}^{m}(k)=\bigcup_{j=0}^{r-1} \Gamma_{r}^{m-1}(k-j) j
$$

where $\Gamma_{r}^{m-1}(k-j) j=\left\{u_{m-1} \ldots u_{1} j \mid \sum_{i=1}^{m-1} u_{i}=k-j\right\}$. Then, the result derives from considering the respective cardinalities.

Moreover, notice that $N_{r}^{m}$ is the number of compositions of $k$ into $m$ parts, each of them being in $\{0,1, \ldots, r-1\}$. (See Fig. 3.) This is equivalent to consider the number of compositions of $k+m$ into $m$ parts, where each part takes values in $\{1,2, \ldots, r\}$. The generating function for these numbers is

$$
G_{r}^{m}(z)=\sum_{k=0}^{(r-1) m} N_{r}^{m}(k) z^{k+m}=\left(z \frac{1-z^{r}}{1-z}\right)^{m}
$$

(see [11] for more details). For instance, for the case $r=m=3$, we get

$$
\left(z \frac{1-z^{3}}{1-z}\right)^{3}=z^{3}+3 z^{4}+6 z^{5}+7 z^{6}+6 z^{7}+3 z^{8}+z^{9}
$$

whose coefficients correspond to the 4 th row in Fig. 3. So that, in particular, $N_{3}^{3}(2)=$ $N_{3}^{3}(4)=6$ (see Fig. 4).

\subsection{Automorphism group}

With respect to the symmetries of the $r$-adic hypertree, notice that from Proposition 3.1, all the eigenvalues of $T_{r}^{m}$ are distinct. As it was shown in [15], this fact has some structural consequences, such as the Abelianity of the automorphism group. Indeed, in what follows we prove that the automorphism group of $T_{r}^{m}$ does not depend on $r$ and is equal to the symmetric group $S_{2}$. This result is a generalization of the case $r=2$ (see [2]).

Proposition 4.2 For each $m \geq 1$ the automorphism group of $T_{r}^{m}$ is the symmetric group $S_{2}$.

Proof. Let $\phi: T_{r}^{m} \rightarrow T_{r}^{m}$ be defined as $\phi\left(u_{m-1} \boldsymbol{w}\right)=\bar{u}_{m-1} \boldsymbol{w}$ for every $\boldsymbol{w} \in \mathbb{Z}_{r}^{m-1}$ (recall that $\left.\bar{u}_{m-1}:=r-1-u_{m}\right)$. We claim that $\operatorname{Aut}\left(T_{r}^{m}\right)=\{I d, \phi\}$, so that $\phi$ is the only non-trivial automorphism of $T_{r}^{m}$. 


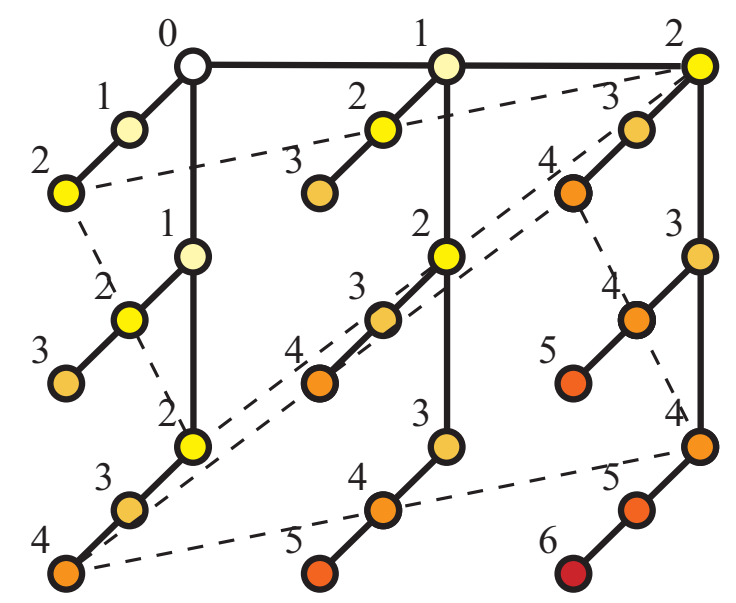

Figure 4: Vertex distances from the root in $T_{3}^{3}$ (dashed lines join the vertices at distance 2 and at distance 4$)$.

Let us first show that $\phi$ is an automorphism. From its definition, it is clear that $\phi$ is an involutive bijection, that is, $\phi(\phi(\boldsymbol{u}))=\boldsymbol{u}$ for every vertex $\boldsymbol{u}$ of $T_{m}$. Now, let $\boldsymbol{u}$ and $\boldsymbol{v}$ be two vertices of $T_{m}$. We have to show that, if $\boldsymbol{u} \sim \boldsymbol{v}$, then $\phi(\boldsymbol{u}) \sim \phi(\boldsymbol{v})$. With this aim assume, without loss of generality, that $\boldsymbol{u}$ is of the form $\boldsymbol{u}=\boldsymbol{w} \alpha 0 \ldots 0$, where either the sequences $\boldsymbol{w}$ or $00 \ldots 0$ can be possibly void and $0 \leq \alpha<r / 2$. Then, we distinguish two cases:

- If $\boldsymbol{u}=\boldsymbol{w} 0 \ldots 0(\alpha=0)$ and $\boldsymbol{v}=\boldsymbol{w} 10 \ldots 0$, then

$$
\phi(\boldsymbol{u})=\boldsymbol{w}(r-1) 0 \ldots 0 \sim \boldsymbol{w}(r-2) 0 \ldots 0=\phi(\boldsymbol{v}) .
$$

- If $\boldsymbol{u}=\boldsymbol{w} \alpha 0 \ldots 0(\alpha \neq 0)$ and $\boldsymbol{v}=\boldsymbol{w}(\alpha \pm 1) 0 \ldots 0$, then

$$
\phi(\boldsymbol{u})=\boldsymbol{w}(r-1-\alpha) 0 \ldots 0 \sim \boldsymbol{w}(r-1-\alpha \mp 1) 0 \ldots 0=\phi(\boldsymbol{v}) .
$$

Now, we prove that $\phi$ is the only non-trivial automorphism of $T_{r}^{m}$ by using induction on $m$. For $m=1, T_{r}^{m}=P_{r}$, and $\operatorname{Aut}\left(P_{r}\right)=S_{2}$. Let $m>1$. As mentioned above (Lemma 2.1 ), the vertex sets $V_{\beta}=\left\{\beta \boldsymbol{w} \mid \boldsymbol{w} \in \mathbb{Z}_{2}^{m-1}\right\}, \beta=0,1, \ldots r-1$, induce $r$ disjoint subgraphs $G_{\beta}=G\left[V_{\beta}\right]$ of $T_{r}^{m}$ isomorphic to $T_{r}^{m-1}$. Assume that $\operatorname{Aut}\left(T_{r}^{m-1}\right)=\{I d, \phi\}$, and let $\gamma$ be an automorphism of $T_{r}^{m}$. Because of the degree sequence of $T_{r}^{m}$, one of the two following cases hold:

- $\gamma(\beta 0 \stackrel{m-1}{\circ} 0)=\beta 0 \stackrel{m-1}{\cdots} 0$,

- $\gamma(\beta 0 \stackrel{m-1}{\cdots} 0)=\bar{\beta} 0 \stackrel{m-1}{\cdots} 0$,

for every $0 \leq \beta \leq r-1$. In the first case, $\gamma$ maps each subgraph $G_{\beta}$ into itself. Moreover, the induced automorphisms let the root fixed. Hence, by induction hypothesis, $\gamma=I d$. In the second case, $\gamma$ maps isomorphically every $G_{\beta}$ into $G_{\bar{\beta}}$. For every $\boldsymbol{w} \in \mathbb{Z}_{r}^{m-1}$, we define the mappings $\gamma_{\beta}$ in the following way:

- If $\gamma(\beta \boldsymbol{w})=\bar{\beta} \boldsymbol{z}$, then $\gamma_{\beta}(\boldsymbol{w})=\boldsymbol{z}$.

Since $\gamma(\beta \mathbf{0})=\bar{\beta} \mathbf{0}$, we have that $\gamma_{\beta}(\mathbf{0})=\mathbf{0}$ and thus $\gamma_{\beta}$ is an automorphism of $T_{r}^{m-1}$ that let the root fixed. Then, by induction hypothesis, $\gamma_{\beta}=I d$, which implies that $\gamma=\phi$. This completes the proof. 


\section{Spectral properties of the $r$-adic hypertree}

This section deals with the spectral properties, eigenvalues and eigenvectors, of the $r$-adic hypertree. The obtained results generalize those given in [3] for the binomial hypertree.

\subsection{Eigenvalues}

In studying the spectrum of the $r$-adic hypertrees, we have to distinguish the cases of $r$ odd and $r$ even. In the first case we have that the spectrum of $T_{r}^{m}$ contains all the other spectra of $T_{r}^{\mu}$ for $\mu<m$, as the following result shows.

Lemma 5.1 If $r$ is odd, the set of eigenvalues of $T_{r}^{m}$ is contained in the set of eigenvalues of $T_{r}^{m+1}$ :

$$
\operatorname{sp} T_{r}^{m} \subset \operatorname{sp} T_{r}^{m+1}
$$

for every $m \geq 0$.

Proof. By induction on $m$. For $m=0, T_{r}^{0}=K_{1}$ has only the eigenvalue 0 and $T_{r}^{1}=K_{1} \sqcap P_{r}=P_{r}$ has also the eigenvalue 0 because $r$ is odd. Suppose that the result holds for a given $m, \operatorname{sp} T_{r}^{m} \subset \operatorname{sp} T_{r}^{m+1}$. Then, by using (6) we get the following implications:

$$
\lambda \in \operatorname{sp} T_{r}^{m+1} \Leftrightarrow \Phi_{r}(\lambda) \in \operatorname{sp} T_{r}^{m} \Rightarrow \Phi_{r}(\lambda) \in \operatorname{sp} T_{r}^{m+1} \Leftrightarrow \lambda \in \operatorname{sp} T_{r}^{m+2},
$$

which completes the proof.

In the case of even $r$, the sets $\left\{\operatorname{sp} T_{r}^{m} \mid m \geq 0\right\}$ are pairwise disjoint. That is, the eigenvalues of the $r$-adic hypertrees of all possible dimensions are all distinct, as the next lemma shows.

Lemma 5.2 If $r$ is even, then for any pair of sequences $i \in \mathbb{Z}_{r}^{s}, \boldsymbol{j} \in \mathbb{Z}_{r}^{t}$,

$$
\boldsymbol{i}=\boldsymbol{j} \quad \Leftrightarrow \quad \lambda_{\boldsymbol{i}}=\lambda_{\boldsymbol{j}}
$$

Proof. Let us assume that $r$ is even. The sufficiency is evident. With respect to the necessity, assume that we have $\lambda_{\boldsymbol{i}}=\lambda_{\boldsymbol{j}}$, for $\boldsymbol{i}=i_{s-1} i_{s-2} \ldots i_{1} i_{0}$ and $\boldsymbol{j}=j_{t-1} j_{t-2} \ldots j_{1} j_{0}$. Hence,

$$
f_{i_{s-1}}\left(f_{i_{s-2}} \circ \cdots \circ f_{i_{1}} \circ f_{i_{0}}(0)\right)=f_{j_{t-1}}\left(f_{j_{t-2}} \circ \cdots \circ f_{j_{1}} \circ f_{j_{0}}(0)\right) .
$$

Then, since according to $(9), f_{h}(x) \in I_{h}$ for any $h \in\{0,1, \ldots, r-1\}$ and $x \in \mathbb{R}$, it must be $i_{s-1}=j_{t-1}$ and hence, $f_{i_{s-2}} \circ \cdots \circ f_{i_{1}} \circ f_{i_{0}}(0)=f_{j_{t-2}} \circ \cdots \circ f_{j_{1}} \circ f_{j_{0}}(0)$. Following the same reasoning we get $i_{s-2}=j_{t-2}$ and $f_{i_{s-3}} \circ \cdots \circ f_{i_{1}} \circ f_{i_{0}}(0)=f_{j_{t-3}} \circ \cdots \circ f_{j_{1}} \circ f_{j_{0}}(0)$, and so on.

Consequently, we only need to show that $s=t$. Assume without loss of generality that $s>t$. Then, applying $t$ times the above process we have $i_{s-1}=j_{t-1}, i_{r-2}=j_{t-2}, \ldots$, $i_{s-t}=j_{0}$ and $f_{i_{s-t-1}} \circ \cdots \circ f_{i_{1}} \circ f_{i_{0}}(0)=0$. This contradicts the fact that, for $r$ even and for every $h$ and $x, f_{h}(x) \neq 0$, and completes the proof.

Notice that Lemma 5.1 implies that the above lemma is not satisfied for $r$ odd.

As $T_{r}^{m}$ is clearly bipartite, its eigenvalue mesh is symmetric [4] and hence, for every $i \in \mathbb{Z}_{r}^{m}$,

$$
\lambda_{\boldsymbol{i}}=-\lambda_{\overline{\boldsymbol{i}}},
$$

where, as before $\bar{\imath}=\bar{\imath}_{m-1} \ldots \bar{\imath}_{1} \bar{\imath}_{0}$. For instance, Fig. 5 shows the spectra of the 3 -adic hypertrees $T_{3}^{m}$ for the cases $0 \leq m \leq 3$, and how each eigenvalue of $T_{3}^{m-1}$ gives rise to three eigenvalues of $T_{3}^{m}$. 


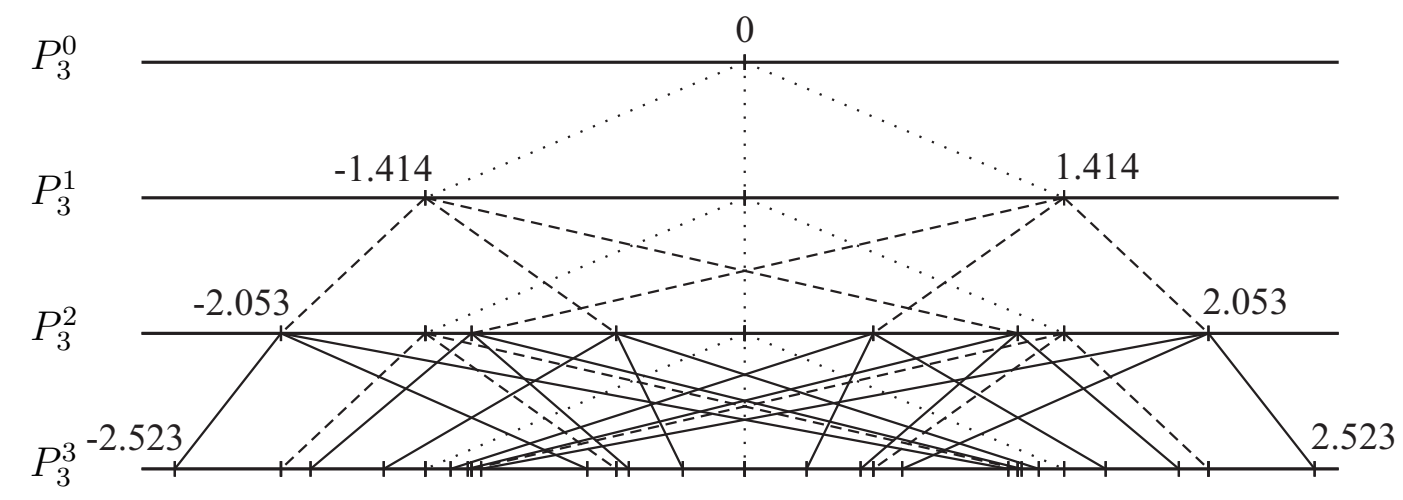

Figure 5: The distribution of the eigenvalues of $P_{3}^{m}$ for $0 \leq m \leq 3$.

The following two results deal with the asymptotic behaviour, as $m \rightarrow \infty$, of the maximum and the minimum (positive) eigenvalues of $T_{r}^{m}$. We show that, while the maximum eigenvalues always behave the same (independently of $r$ ), the asymptotic decreasing rate of the minimum positive eigenvalue follows a power law for $r$ even and an exponential law for $r$ odd. (See Fig. 6.)

Proposition 5.3 For every fixed $k \geq 1$, let $\lambda_{r^{m}-k}$ be the $k$-th largest eigenvalue of $T_{r}^{m}$. Its asymptotic behavior is

$$
\lambda_{r^{m}-k} \sim \sqrt{2 m}
$$

for any value of $r$.

Proof. For simplicity of notation, let $\lambda[m]=\lambda_{r^{m}-k}$. Then, by the proof of Proposition 3.1, $\lambda[m]$ is the largest solution of the equation $\Phi_{r}(x)=\lambda[m-1]$. (See Fig. 6.) By using (7), the asymptotic behaviour of $\Phi_{r}(x)$, as $x \rightarrow \infty$, is

$$
\Phi_{r}(x)=x-\frac{\phi_{r-2}(x)}{\phi_{r-1}(x)} \sim x-\frac{1}{x},
$$

since all the involved characteristic polynomials are monic. Hence, we have that $x \sim \lambda[m]$ iff $x-\frac{1}{x} \sim \lambda[m-1]$. Then, we only need to check that, $x=\sqrt{2 m}$ satisfies $x-\frac{1}{x} \sim$ $\sqrt{2(m-1)}$, when $m \rightarrow \infty$. Indeed, $\left(\sqrt{2 m}-\frac{1}{\sqrt{2 m}}\right)^{2}=2 m-2+\frac{1}{2 m} \sim 2(m-1)$.

Proposition 5.4 The asymptotic behaviour of the minimum positive eigenvalue of $T_{r}^{m}$ is

$$
\begin{array}{rlrl}
\lambda_{\frac{r m+1}{2}} & \sim\left(\frac{r+1}{2}\right)^{-m} & (r \text { odd }) \\
\lambda_{\frac{r m}{2}} \sim \frac{r \sqrt{2}}{\sqrt{m}} & (r \text { even }) .
\end{array}
$$

Proof. First, let $\phi_{r}(x)=a_{0, r}+a_{1, r} x+a_{2, r} x^{2}+\cdots$, where the coefficients $a_{0, r}, a_{1, r}$, $a_{2, r}$ can be computed by using (4) and turn out to be

$$
\begin{aligned}
& a_{0, r}=(-1)^{\frac{r}{2}}, \quad a_{2, r}=(-1)^{\frac{r-2}{2}} \frac{r^{2}+2 r}{8} \quad(r \text { even }) ; \\
& a_{1, r}=(-1)^{\frac{r-1}{2}} \frac{r+1}{2} \quad(r \text { odd }),
\end{aligned}
$$




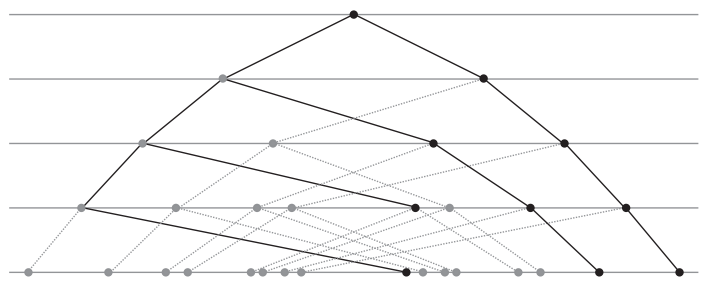

(a)

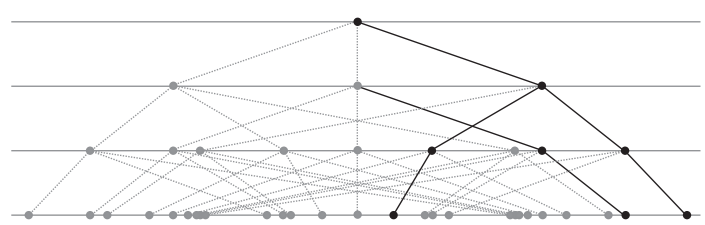

(b)

Figure 6: The maximum and the minimum eigenvalues in the cases (a) $r$ even and (b) $r$ odd.

the other cases being zero.

For $r$ odd, $\lambda\{m\}=\lambda_{\frac{r m+1}{2}}$ is the smallest positive solution of the equation $\Phi_{r}(x)=$ $\lambda\{m-1\}$. (See Fig. 6 (b).) The behaviour of $\Phi_{r}(x)$, as $x \rightarrow 0$, is

$$
\Phi_{r}(x)=\frac{\phi_{r}(x)}{\phi_{r-1}(x)} \sim \frac{a_{1, r} x}{a_{0, r}},
$$

which, using (19) and (20), gives

$$
\Phi_{r}(x) \sim \frac{(-1)^{\frac{r-1}{2}} \frac{r+1}{2} x}{(-1)^{\frac{r-1}{2}}}=\frac{r+1}{2} x .
$$

Hence, we have that $x \sim \lambda_{m}$ iff $\frac{r+1}{2} x \sim \lambda_{m-1}$. This implies

$$
x \sim \lambda_{m} \sim\left(\frac{r+1}{2}\right)^{-m}
$$

as claimed in (17).

For $r$ even, $\lambda\{m\}=\lambda_{\frac{r}{2} m}$ is now the smallest positive solution of the equation $\Phi_{r}(x)=$ $-\lambda[m-1]$. (See Fig. 6 (a).) Besides, the asymptotic behaviour of $\Phi_{r}(x)$, as $x \rightarrow 0$, is

$$
\Phi_{r}(x)=\frac{\phi_{r}(x)}{\phi_{r-1}(x)} \sim \frac{a_{2, r} x^{2}+a_{0, r}}{a_{1, r-1} x} .
$$

Then, by using again (19) and (20), we get the equation

$$
\frac{(-1)^{\frac{r-2}{2}} \frac{r^{2}+2 r}{8} x^{2}+(-1)^{\frac{r}{2}}}{(-1)^{\frac{r-2}{2}} \frac{r}{2} x}=-\lambda[m-1] \sim-\sqrt{2(m-1)} .
$$

That is

$$
\left(r^{2}+2 r\right) x^{2}+4 r \sqrt{2(m-1)} x-8 \sim 0 .
$$

Solving for $x$, the positive solution is (after some simplifications)

$$
x \sim \frac{2 \sqrt{2}}{\sqrt{r^{2} m+2 r}+r \sqrt{m+1}} \sim \frac{\sqrt{2}}{r \sqrt{m}},
$$

as claimed in (18).

The following result is a consequence of the fact that $\lambda_{\alpha} \dot{i}, \alpha=0,1, \ldots, r-1$, are the roots of the $r$-th degree polynomial $p_{r}(x)=\phi_{r}(x)-\lambda_{\boldsymbol{i}} \phi_{r-1}(x)=0$. 
Lemma 5.5 For every $i \in \mathbb{Z}_{r}^{m-1}$ we have

$$
\begin{aligned}
& \sum_{\alpha=0}^{r-1} \lambda_{\alpha} \boldsymbol{i}=\lambda_{\boldsymbol{i}}, \\
& \prod_{\alpha=0}^{r-1} \lambda_{\alpha}= \begin{cases}(-1)^{r / 2} & (r \text { even }), \\
(-1)^{(r-1) / 2} \lambda_{i} & (r \text { odd }) .\end{cases}
\end{aligned}
$$

Proof. Recall that the $r$-degree Chebyshev polynomial is even (respectively, odd) if $r$ is even (respectively, odd). Then, the coefficient of $x^{r-1}$ in $p_{r}(x)$ is $\lambda_{\boldsymbol{i}}$ and this gives (21).

Moreover, the constant term of $p_{r}(x)$ is

$$
p_{r}(0)=\phi_{r}(0)-\lambda_{i} \phi_{r-1}(0)=(-1)^{r} \prod_{\alpha=0}^{r-1} \lambda_{\alpha} \boldsymbol{i}
$$

where $\phi_{0}(0)=1, \phi_{1}(0)=0$ and $\phi_{r}(0)=-\phi_{r-2}(0), r \geq 2$, by (4). This yields $\phi_{r}(0)=$ $(-1)^{r / 2}$ for $r$ even and $\phi_{r}(0)=0$ for $r$ odd, as expected. Then, substituting these values into (23), we obtain (22).

Adding up for every $i \in \mathbb{Z}_{r}^{m-1}$ we get the following corollary.

Corollary 5.6 The eigenvalues of the r-adic hypertrees satisfy the following properties:

$$
\begin{aligned}
\sum_{\boldsymbol{i} \in \mathbb{Z}_{r}^{m}} \lambda_{\alpha i} & =\sum_{\boldsymbol{i} \in \mathbb{Z}_{r}^{m-1}} \lambda_{\alpha} \boldsymbol{i}=\cdots=0, \\
\sum_{\boldsymbol{i} \in \mathbb{Z}_{r}^{m-1}} \prod_{\alpha=0}^{r-1} \lambda_{\alpha} \boldsymbol{i} & = \begin{cases}(-1)^{r / 2} r^{m-1} & (r \text { even }), \\
0 & (r \text { odd }) .\end{cases}
\end{aligned}
$$

\subsection{Eigenvectors}

The eigenvectors of $T_{r}^{m}$ can be recursively computed by using Proposition 3.3. For instance, for $r=3$, we can start from $T_{3}^{0}=K_{1}$, with eigenvalue 0 and eigenvector $\boldsymbol{u}=1$. Then, we need to consider the eigenvectors of the matrix

$$
\boldsymbol{A}(0)=\left(\begin{array}{lll}
0 & 1 & 0 \\
1 & 0 & 1 \\
0 & 1 & 0
\end{array}\right)
$$

(note that it corresponds to the adjacency matrix of the path $P_{3}$ ), with eigenvalues $\lambda_{0}=$ $-\sqrt{2}, \lambda_{1}=0$ and $\lambda_{2}=\sqrt{2}$. Then, by (12), their respective eigenvectors are:

$$
\begin{aligned}
& \boldsymbol{w}_{0}^{0}=\left(\phi_{2}\left(\lambda_{0}\right), \phi_{1}\left(\lambda_{0}\right), \phi_{0}\left(\lambda_{0}\right)\right)^{\top}=(1,-\sqrt{2}, 1)^{\top}, \\
& \boldsymbol{w}_{1}^{0}=\left(\phi_{2}\left(\lambda_{1}\right), \phi_{1}\left(\lambda_{1}\right), \phi_{0}\left(\lambda_{1}\right)\right)^{\top}=(-1,0,1)^{\top}, \\
& \boldsymbol{w}_{2}^{0}=\left(\phi_{2}\left(\lambda_{2}\right), \phi_{1}\left(\lambda_{2}\right), \phi_{0}\left(\lambda_{2}\right)\right)^{\top}=(1, \sqrt{2}, 1)^{\top},
\end{aligned}
$$

since $\phi_{2}=x^{2}-1, \phi_{1}=x$ and $\phi_{0}=1$. From the above proposition, these are precisely the eigenvectors of $T_{3}^{1}=P_{3}$, since $\boldsymbol{w}_{h}^{0} \otimes \boldsymbol{u}=\boldsymbol{w}_{h}^{0}$, for $h=0,1,2$ (as expected). In general, we have the following result: 
Proposition 5.7 For every sequence $\boldsymbol{i}=i_{m-1} i_{m-2} \ldots i_{0} \in \mathbb{Z}_{r}^{m}$, the r-adic hypertree $T_{r}^{m}$ has the eigenvalue

$$
\lambda_{i}=f_{i_{m-1}} \circ f_{i_{m-2}} \circ \cdots \circ f_{i_{0}}(0)
$$

with corresponding eigenvector

$$
\begin{aligned}
\boldsymbol{u}_{\boldsymbol{i}} & =\boldsymbol{u}_{i_{m-1} i_{m-2} \ldots i_{1} i_{0}} \\
& =\boldsymbol{w}_{i_{m-1} i_{m-2} \ldots i_{1} i_{0}} \otimes \boldsymbol{w}_{i_{m-2} i_{m-3} \ldots i_{1} i_{0}} \otimes \cdots \otimes \boldsymbol{w}_{i_{1} i_{0}} \otimes \boldsymbol{w}_{i_{0}}
\end{aligned}
$$

where, for any $0 \leq s \leq m-1$, the vectors $\boldsymbol{w}_{\boldsymbol{j}}=\boldsymbol{w}_{i_{s-1} i_{s-2} \ldots i_{1} i_{0}}$ are seen as $r \times 1$ matrices and they are computed according (12), that is

$$
\boldsymbol{w}_{\boldsymbol{j}}=\left(\phi_{r-1}\left(\lambda_{\boldsymbol{j}}\right), \phi_{r-2}\left(\lambda_{\boldsymbol{j}}\right), \ldots, \phi_{1}\left(\lambda_{\boldsymbol{j}}\right), \phi_{0}\left(\lambda_{\boldsymbol{j}}\right)^{\top}\right.
$$

Proof. Since $T_{r}^{m}=T_{r}^{m-1} \sqcap P_{r}$, the result follows by applying recursively Proposition 3.3 .

For instance, the hypertree $T_{3}^{2}$ has eigenvalues (numbers are given with three decimal places)

$$
\begin{aligned}
& \lambda_{00}=-2.052, \lambda_{01}=-1.414, \lambda_{02}=-1.208, \lambda_{10}=-0.569, \quad \lambda_{11}=0, \\
& \lambda_{12}=0.569, \quad \lambda_{20}=1.208, \lambda_{21}=-1.414, \lambda_{22}=2.053 .
\end{aligned}
$$

with respective eigenvectors

$$
\boldsymbol{u}_{i_{1} i_{0}}=\boldsymbol{w}_{i_{1} i_{0}} \otimes \boldsymbol{w}_{i_{0}}=\left(\begin{array}{c}
\phi_{2}\left(\lambda_{i_{1} i_{0}}\right) \phi_{2}\left(\lambda_{i_{0}}\right) \\
\phi_{2}\left(\lambda_{i_{1} i_{0}}\right) \phi_{1}\left(\lambda_{i_{0}}\right) \\
\phi_{2}\left(\lambda_{i_{1} i_{0}}\right) \phi_{0}\left(\lambda_{i_{0}}\right) \\
\phi_{1}\left(\lambda_{i_{1} i_{0}}\right) \phi_{2}\left(\lambda_{i_{0}}\right) \\
\phi_{1}\left(\lambda_{i_{1} i_{0}}\right) \phi_{1}\left(\lambda_{i_{0}}\right) \\
\phi_{1}\left(\lambda_{i_{1} i_{0}}\right) \phi_{0}\left(\lambda_{i_{0}}\right) \\
\phi_{0}\left(\lambda_{i_{1} i_{0}}\right) \phi_{2}\left(\lambda_{i_{0}}\right) \\
\phi_{0}\left(\lambda_{i_{1} i_{0}}\right) \phi_{1}\left(\lambda_{i_{0}}\right) \\
\phi_{0}\left(\lambda_{i_{1} i_{0}}\right) \phi_{0}\left(\lambda_{i_{0}}\right)
\end{array}\right)
$$

where $i_{1}, i_{0} \in \mathbb{Z}_{3}$ and, recalling the above eigenvalues of $T_{3}^{1}=P_{3}, \lambda_{0}=-\sqrt{2}, \lambda_{1}=0$ and $\lambda_{2}=\sqrt{2}$.

\section{Acknowledgments}

Research supported by the Education and Science Ministry, Spain, and the European Regional Development Fund under projects MTM2005-08990-C02-01 and TEC2005-03575 and by the Catalan Research Council under project 2005SGR00256. 


\section{References}

[1] R. Albert and A.L. Barabási, Statistical mechanics of complex networks, Rev. Modern Phys. 74 (2002), 47-97.

[2] L. Barrière, F. Comellas, C. Dalfó, M.A. Fiol, The hierarchical product of graphs, Discrete Appl. Math., submitted, http://hdl.handle.net/2117/672.

[3] L. Barrière, F. Comellas, C. Dalfó, M.A. Fiol, On the spectra of hypertrees, Linear Algebra Appl., submitted, http://hdl.handle.net/2117/891.

[4] N. Biggs, Algebraic Graph Theory, Cambridge University Press, Cambridge, 1974, second edition, 1993.

[5] G. Chartrand and L. Lesniak, Graphs \& Digraphs, third edition, Chapman and Hall, London, 1996.

[6] F.R.K. Chung, Diameters and eigenvalues, J. Amer. Math. Soc. 2 (1989) 187-196.

[7] T.H. Cormen, C.E. Leiserson, R.L. Rivest, C. Stein, Introduction to Algorithms, MIT Press, Cambridge, Massachussets, 1990, second edition, 2001.

[8] D.M. Cvetković, M. Doob, H. Sachs, Spectra of Graphs. Theory and Applications, third edition, Johann Ambrosius Barth, Heildelberg, 1995.

[9] M.A. Fiol, Eigenvalue interlacing and weight parameters of graphs, Linear Algebra Appl. 290 (1999), 275-301.

[10] M.A. Fiol, Algebraic characterizations of distance-regular graphs, Discrete Math. 246 (2002), 111-129.

[11] P. Flajolet, R. Sedgewick, Analytic Combinatorics, 2007. http://algo.inria.fr/flajolet/Publications/books.html

[12] C. D. Godsil, Algebraic Combinatorics, Chapman and Hall, New York, 1993.

[13] W.H. Haemers, Interlacing eigenvalues and graphs, Linear Algebra Appl. 226-228 (1995), 593-616.

[14] S.M. Hedetniemi, S.T. Hedetniemi and A. Liestman, A survey of gossiping and broadcasting in communication networks, Networks 18 (1988), 319-349.

[15] A. Mowshowitz, The group of a graph whose adjacency matrix has all distinct eigenvalues, in F. Harary ed., Proof Techniques in Graph Theory, Academic Press, New York, 1969, pp. 109-110.

[16] A.J. Schwenk, Computing the characteristic polynomial of a graph, Graphs and Combin., Proc. Capital Conf., Washington, D.C. 1973, Lect. Notes Math. 406 (1974), $153-172$.

[17] J. R. Silvester, Determinants of block matrices, Math. Gaz. 84 (2000), 460-467.

[18] G. Szegö, Orthogonal Polynomials, fourth edition. American Mathematical Society, Colloquium Publications, Vol. XXIII. American Mathematical Society, Providence, R.I., 1975. 